\title{
Targeted re-sequencing of coding DNA sequences for SNP discovery in non-model species
}

Daniel W. Förster ${ }^{1, *}$, James K. Bull ${ }^{1}$, Dorina Lenz ${ }^{1}$, Marijke Autenrieth ${ }^{1,2}$, Johanna L. A. Paijmans ${ }^{2}$, Robert H. S. Kraus ${ }^{3,4}$, Carsten Nowak ${ }^{5}$, Helmut Bayerl ${ }^{6}$, Ralph Kühn ${ }^{6}$, Alexander P. Saveljev ${ }^{7}$, Magda Sindičić $^{8}$, Michael Hofreiter ${ }^{2}$, Krzysztof Schmidt ${ }^{9}$, Jörns Fickel ${ }^{1,2}$

\section{Author affiliations:}

${ }^{1}$ Department of Evolutionary Genetics, Leibniz-Institute for Zoo and Wildlife Research, AlfredKowalke-Str. 17, D-10315 Berlin, Germany

${ }^{2}$ Institute for Biochemistry and Biology, University of Potsdam, Karl-Liebknecht-Str. 24-25, D-14476 Potsdam, Germany

${ }^{3}$ University of Konstanz, Department of Biology, 78457 Konstanz, Germany

${ }^{4}$ Max Planck Institute for Ornithology, Department of Migration and Immuno-Ecology, Am Obstberg 1, D-78315 Radolfzell, Germany

${ }^{5}$ Conservation Genetics Group, Senckenberg Research Institute and Natural History Museum Frankfurt, D-63571 Gelnhausen, Germany

${ }^{6}$ Molecular Zoology Unit, Research Department Animal Sciences, Technical University Munich, HansCarl-von-Carlowitz-Platz 2, D-85354 Freising, Germany

${ }^{7}$ Russian Research Institute of Game Management and Fur Farming, Department of Animal Ecology, Preobrazhenskaya str. 79, 610000 Kirov, Russia

${ }^{8}$ Faculty of Veterinary Medicine, University of Zagreb, Heinzelova ul. 68, 10000 Zagreb, Croatia

${ }^{9}$ Mammal Research Institute, Polish Academy of Sciences, 17-230 Białowieza, Poland

* Author to whom correspondences should be addressed: Daniel W. Förster, dwgfoerster@gmail.com Department of Evolutionary Genetics, Leibniz-Institute for Zoo and Wildlife Research, AlfredKowalke-Str. 17, D-10315 Berlin, Germany

Fax.: +49 (0) 305126104 
Keywords: CDS, conservation genetics, Eurasian lynx, genetic monitoring, hybridization capture, singlenucleotide polymorphism

Running title: CDS enrichment for SNP discovery

\section{ABSTRACT}

Hybridization capture coupled with high-throughput sequencing can be used to gain information about nuclear sequence variation at hundreds to thousands of loci. A cross-species approach makes use of molecular data of one species to enrich target loci in other (related) species. This is particularly valuable for non-model organisms, for which often no a priori knowledge exists regarding these loci. Here, we have adopted cross-species capture to obtain data for 809 nuclear coding DNA sequences (CDS) in a non-model organism, the Eurasian lynx Lynx lynx, using baits designed with the help of the published genome of a related model organism (the domestic cat Felis catus). In this manner, we were able to survey intraspecific variation at hundreds of nuclear loci across the European range of L. lynx. A large set of bi-allelic candidate SNPs was then tested in a high throughput SNP-genotyping platform (Fluidigm), which we reduced to a final 96 SNP-panel based on assay performance and reliability; validation was carried out with additional samples not included in the SNP discovery phase. The 96 SNP-panel developed from CDS performed very successfully in the identification of individuals and in population genetic structure inference (incl. the assignment of individuals to their source population). In keeping with recent studies, our results show that genic SNPs can be valuable for genetic monitoring of wildlife species. 


\section{INTRODUCTION}

For studies that require sequence information of only particular parts of a genome, it has become common practice to query nuclear genomes (usually of humans and model-organisms) for genetic variation using 'enrichment' techniques (also called 'hybridization capture'). In this approach, so-called 'baits' (DNA/RNA molecules) with sequences complementary to genomic regions of interest are used to capture said regions in genetic libraries prior to sequencing, while unwanted 'off-target' DNA is washed away (Gasc et al., 2016). This method is so successful that commercial suppliers already offer enrichment assays for a plethora of applications (e.g. targeting loci associated with particular traits or diseases) and also offer to design custom assays. Even automation of the entire process (library building and subsequent enrichment) is already available.

For molecular, ecological and taxonomic research in non-model organisms, this technique has proven to be highly valuable. It has strongly facilitated the study of intraspecific variation of whole mitochondrial genomes, particularly when DNA from sample material is scarce and/or degraded or when the resolution of PCR-amplified single short mitochondrial sequences is insufficient to answer particular research questions (Paijmans et al., 2013). The taxonomically wide accessibility of mtDNA reference sequences for bait design together with the availability of do-it-yourself protocols for bait generation (Maricic et al., 2010) have also facilitated both its implementation and proliferation. Besides its success in mtDNA research, the technique has also been applied to nuclear loci in non-model organisms, but here mainly in interspecific studies to address taxonomic uncertainties. The promiscuity of the baits in the hybridization capture process allows for up to $40 \%$ sequence divergence between bait and target (Li et al., 2013), a property that can be exploited to obtain relatively complete datasets of 100s-1000s loci (Lemmon et al., 2012; Faircloth et al., 2012; Bi et al., 2012; Prum et al., 2015) across different taxonomic levels (genera, families, even orders). 
In this study, we demonstrate the application of hybridization capture for single-nucleotide polymorphism (SNP) discovery in a non-model species and show how the identified SNPs can be utilized for cost-effective genetic monitoring of an elusive carnivore, the Eurasian lynx Lynx lynx.

We used a 'cross-species capture' approach to enrich target loci from our study species (lynx) using baits designed from a fully annotated reference genome of a related species, namely the domestic cat. In order to avoid capturing paralogues, yet have loci evenly distributed throughout the lynx genome, we designed cat baits to target single-copy coding DNA sequences (CDS). To minimize ascertainment bias, lynx samples used for SNP discovery covered the European distribution range of the species, including some reintroduced populations.

In the past, the applicability of SNPs for monitoring wildlife populations was quite limited, because the (usually very low) amount of template DNA extractable from non-invasively collected samples constrained the number of SNP loci that could be genotyped in such samples (e.g. Kraus et al., 2015, and references therein). However, technological advancements such as nanofluidics substantially reduced the required reaction volumes for SNP genotyping, making it possible to simultaneously type numerous SNP loci even from very little template material. For this reason, we aimed to develop a SNP-panel that can be routinely used with the nanofluidic Dynamic Array Chip technology implemented in the Fluidigm platform (Fluidigm Corp., SanFrancisco, CA, USA).

Here, we report the development of a 96 SNP-panel for the Eurasian lynx Lynx lynx (Fig. 1A). We outline how we (i) used the genomic resources available for model organisms to design baits and then enriched target loci in our study species; (ii) filtered the intraspecific variation in our study species for candidate SNP loci; (iii) evaluated a large set of candidate SNPs in our chosen genotyping platform, and (iv) settled on 96 loci for the final SNP-panel. Then, we present how the newly developed SNP-panel performed in the genetic monitoring of our study species using additional samples (not included in the SNP discovery). To evaluate performance, we specifically focused on the ability of a SNP-panel 
developed from coding DNA sequences for (i) individual discrimination and (ii) analysis of genetic population structure, including the correct assignment of individuals to their source population.

\section{MATERIALS AND METHODS}

\section{Biological samples}

DNA was extracted from tissue (liver or muscle) or blood using the commercial First-DNA all tissue kit

(GEN-IAL GmbH, Troisdorf, Germany). 26 Eurasian lynx from four European populations (von Arx et al. 2004) were used in the initial cross-species capture: Baltic - Estonia $(N=2)$, Latvia $(N=3)$, Poland $(N=2)$, Russia $(N=4) ;$ Nordic - Finland $(N=3)$, Norway $(N=3)$; Carpathian - Romania $(N=3)$, Slovakia $(N=2)$; and Dinaric - Croatia $(N=2)$, Slovenia $(N=2)$ (Table 1, Fig. 1B).

Applicability of the final 96 SNP-panel was then assessed by genotyping an additional 96 lynx samples originating from the same populations (below, but see also supplementary Table S1).

The Dinaric population was intentionally sampled as a distinct reintroduced population (originating from the Carpathian population), for the assessment of correct individual assignment (below).

\section{Bait design}

We compared the annotated genomes of the domestic cat (Felis catus v6.2), domestic dog (Canis lupus familiaris v3.1), horse (Equus caballus v2.0), cow (Bos taurus v3.1) and pig (Sus scofa v10.2) using EVOLMARKERS (Li et al., 2012) to identify single-copy protein coding genes present in all of these taxa. In order to avoid paralogues, candidate target loci were restricted to CDS with less than $40 \%$ similarity to intraspecific sequences using a BLAST approach (Li et al., 2012). This restriction ensured that target loci would be unambiguously identifiable. To increase the chance of SNP discovery and facilitate the 
development of assays, we excluded short sequences and thus chose targets with a minimum length of $400 \mathrm{bp}$, which yielded 1357 CDS markers. Potential targets were then further filtered by selecting a single CDS per autosomal gene, reducing the set to 809 CDS markers. As capture was to be performed on a felid species, we used Felis catus CDS to design the baits (Fig. 1C, see also information summary in supplemental Table S2). The custom tailored MYbaits ${ }^{\circledR}$ target enrichment kit (MYcroarray, Ann Arbor, MI, USA) covering all 809 CDS (having a total length of 618547 bp) finally consisted of 8922 biotinylated RNA baits ( $2 \times$ tiling, 120 bp length). The bait design is available as a FASTA file on DRYAD (doi: provided upon acceptance).

\section{Capture of CDS and sequencing}

Illumina sequencing libraries were built following a published DIY protocol (Meyer \& Kircher, 2010) with some modifications reducing both loss of template and costs (Fortes \& Paijmans, 2015).

Libraries were captured individually following suggested modification of the MYbaits protocol (Li et al., 2013). In brief, volumes were reduced, the amount of synthetic RNA-baits used per capture was reduced (8-fold), and the standard hybridization temperature of $65^{\circ} \mathrm{C}$ was replaced by a 'touchdown' protocol with hybridization temperature decreasing from $65^{\circ} \mathrm{C}$ to $50^{\circ} \mathrm{C}$ in $5^{\circ} \mathrm{C}$ increments every $11 \mathrm{hrs}$. Detailed methods for library building and capture can be found in the Appendix.

The library of each individual $(N=26)$ was captured two consecutive times; the eluate from the first capture (i.e. the enriched library) was amplified and used in a second round of capture, as this has been reported to increase the number of on-target reads for both within-species and cross-species capture ( $\mathrm{Li}$ et al., 2013; Templeton et al., 2013). We confirmed this by sequencing the first five samples processed also after the first capture round, observing a four- to seven-fold increase in the number of unique ontarget sequences after two consecutive captures (supplemental Fig. S1). 
Enriched libraries were paired-end sequenced on two Illumina platforms (Illumina, San Diego, CA, USA): MiSeq using v2 300-cycle kits, and NextSeq using v1 150-cycle MidOutput kits.

\section{Data processing}

Pre-Processing: De-multiplexing of paired-end reads using BCL2FASTQ v2.17.1.14 (Illumina, Inc.) was followed by removal of adapter sequences using CUTADAPT v1.3 (Martin, 2011). Adapter-clipped reads were then quality-trimmed using a sliding window approach in TRIMMOMATIC (Bolger et al., 2014), with the phred quality threshold set at $\mathrm{Q}=20$ and window length of $10 \mathrm{bp}$. Overlapping paired-end reads having a minimal overlap of $10 \mathrm{bp}$ were merged using the software FLASH v1.2.8 (Magoč \& Salzberg, 2011). Merged and non-merged sequences were used as input for mapping using BURROWS-WHEELER ALIGNER V0.7.10 (BWA; Li \& Durbin, 2009) with seeding disabled (Schubert et al., 2012).

Reference processing: Because lynx CDS display little sequence divergence from orthologous cat CDS ( $\sim \%$ - 4\% sequence divergence; DWF, unpublished data), we were able to use a simple approach to generate lynx CDS references. First, we retrieved 300bp of flanking sequences (both 5' and 3') of each cat CDS (cat genome v8.0) to extend the cat reference sequence beyond CDS boundaries. This was done by querying the CDS bait sequences vs. the Felis catus v8.0 genome using BLASTN (BLAST+ v2.2.29); the resulting hits were restricted to only one target hit (-max_target_seqs 1) and were further filtered to match only the predicted chromosome. The actual CDS sequence including flanking sequence was retrieved by applying BEDTOOLS getfasta (v2.17.0) using $\pm 300 \mathrm{bp}$ CDS positions on the cat reference. Then, these extended cat sequences (CDS + flanks) served as reference for mapping all lynx sequences (i.e. from all samples). Aligned sequences were de-duplicated using MarkDuplicates from PICARDTOOLS v1.106 (https://github.com/broadinstitute/picard). Variant calling was carried out using SAMTOOLS v1.1 (Li et al., 2009) and BCFTOOLS v1.2 (http://github.com/samtools/bcftools) to determine the most common lynx variant at every position; the cat reference was modified accordingly. For variants 
present at $\geq 3 \times$ and alternate base frequency $>0.5$ the alternate (lynx) base was used to generate the lynx consensus sequence. In this manner, we converted the cat sequence into a lynx 'consensus' sequence for each CDS, which included 300 bp of 5' and 3' flanking sequence; henceforth, the 'lynx CDS reference' (of 1,102,167 bp length). We carried out the same procedure using the recently published Iberian lynx (Lynx pardinus) genome as reference (Abascal et al., 2016), representing a closer relative of our study species, albeit with a less complete genome assembly. It should be noted, however, that in cases where the target species is highly divergent from the reference species, other approaches are advised to generate target species reference sequences (e.g. Yuan et al., 2016; Portik et al., 2016).

Sample Processing: We generated a separate consensus sequence for each lynx sample by mapping against the lynx CDS reference. Following a second round of mapping against this sample-specific consensus sequence (in order to recover as much data as possible), GATK UnifiedGenotyper (v1.6) was then used to identify sequence variants (SNPs and InDels) in the 26 Eurasian lynx samples used for SNP discovery. Of all SNPs identified, only SNPs with a coverage $\geq 15 \times$ were retained as candidates for the SNP panel. These candidate loci were then reduced to 144 SNPs (representing a 50\% surplus over the target number of 96 SNPs for the final panel) based on the following criteria: (i) SNPs had to be biallelic (a requirement of the genotyping platform); (ii) minor allele frequency should be greater than $10 \%$ (to avoid loci with rare alleles and hence potentially low applicability); (iii) no variants (SNPs or InDels) within $100 \mathrm{bp}$ of the candidate SNP to avoid interference in the genotyping process; (iv) only one SNP allowed per CDS to avoid physical linkage; (v) SNPs should be as widely distributed across the genome as possible (using the cat genome as reference, Fig. 1c); and (vi) SNPs should not lay in the flanking regions of the CDS, because our goal was to create a SNP panel from data obtained following crossspecies enrichment of CDS (flanking sequences may not be available for all study species). 
We aimed to generate a lynx SNP-panel with 96 SNPs for high-throughput genotyping using Fluidigm's SNPtype $^{\mathrm{TM}}$ assays (Fluidigm Corp., SanFrancisco, CA, USA). Specifically, we intended to use the '96.96 Dynamic Array Chip for Genotyping' that allows the simultaneous genotyping of 96 samples at 96 bi-allelic SNPs, which is particularly useful when only little sample material is available. The latter was an important criterion for platform selection because non-invasively collected samples represent an important resource for genetic monitoring of wildlife populations, and these often yield very little DNA for analysis, which thus needs to be used very efficiently. The nanofluidic Dynamic Array Chip technology employed in this platform reduces PCR volumes to nanolitres, performing 9216 (96×96) single-plex reactions in a highly automated fashion. As little as $1.25 \mu \mathrm{L}$ of DNA extract $(0.5 \mathrm{ng} / \mu \mathrm{L})$ is sufficient to genotype one sample at 96 SNPs. Genotyping itself is accomplished using allele-specific primers labeled with fluorescent dyes. For samples with low amounts of template (e.g. non-invasively collected samples) a pre-amplification is strongly recommended (Nussberger et al., 2014; Kraus et al., 2015).

Prior to the selection of 96 SNPs for the final genotyping panel we pre-selected 144 candidate SNPs (96 +48 ) for evaluation (above), of which we randomly chose $15(10.4 \%)$ for verification in five of the 26 Eurasian lynx samples using Sanger sequencing. After their successful verification, we designed SNPtype $^{\mathrm{TM}}$ assays for all 144 SNPs.

Using previously established procedures (Kraus et al., 2015) we assessed genotyping errors for all 144 SNPtype ${ }^{\mathrm{TM}}$ assays. Specifically, we genotyped the 26 Eurasian lynx samples that had been sequenced for SNP discovery using both undiluted $(50 \mathrm{ng} / \mu \mathrm{L})$ and diluted $(0.5 \mathrm{ng} / \mu \mathrm{L})$ samples. The latter served to approximate poor DNA quality samples (e.g. coming from non-invasively collected samples). We used 'genotyping treatment c2' (Kraus et al., 2015; less dilution of specific target amplification [STA] products, 42 cycles of amplification), and evaluated (by locus) the following properties: genotype 
consistency across dilutions, the incidences of missing data, and genotype consistency with the Illumina sequencing data.

For the assembly of the final 96 SNP-panel, we chose the assays with the most consistent genotyping performance across the 26 lynx samples (i.e. showed lowest rates of missing data and genotyping errors) and for which the three genotypes $(\mathrm{AA}, \mathrm{AB}, \mathrm{BB})$ could be unambiguously distinguished in the scatter plots generated as output by the EP1 genotyping software (Fluidigm Corp.). To test the general applicability of the final 96 SNP-panel, we genotyped a set of 96 Eurasian lynx samples from across Europe (Table S1), none of which had been used in the SNP discovery. Again, we randomly chose 10 out of 96 loci (10.4\%) to be verified by Sanger sequencing in five out of the 96 Eurasian lynx.

As DNA of prey species may contaminate DNA extracted from non-invasively collected fecal samples, we also performed cross-species testing of the SNP-panel on typical representatives of common prey taxa: roe deer Capreolus capreolus, European red deer Cervus elaphus, European hare Lepus europaeus, house mouse Mus musculus, pine marten Martes martes, and American mink Neovison vison.

\section{Individual identification}

We used two approaches to examine the power of the newly designed 96 SNP-panel to discriminate among individuals. Firstly, we used GIMLET v.1.3.3 (Valière, 2002) to estimate (across loci) both the unbiased 'probability of identity' ( $\mathrm{PID}_{\mathrm{unb}}$ ) and the more conservative 'probability of identity given siblings' $\left(\mathrm{PID}_{\mathrm{sib}}\right)$. These probabilities were estimated separately for each population with at least 10 individuals (Estonia, Latvia, Poland, Norway, Slovenia), as well as for the genetic clusters identified in the population structure analyses (below).

Secondly, we directly examined the performance of the SNPs to differentiate all individuals in our data set. Specifically, we examined how well different subsets of loci performed for identifying individuals. 
We examined a range of subset sizes $(10,20,30,40,50,60,70,80$, and 90 loci), and examined the results for 10,000 permutations per subset size; for this, subsets of loci were randomly drawn from the entire dataset without replacement. This analysis was conducted in the statistical programming environment R (http://www.cran.r-project.org) using a custom script (deposited on DRYAD under doi: provided upon acceptance).

\section{Population structure inferences}

For the assessment of SNP performance regarding the detection of genetic substructure and population assignment we used two common methods: principal component analysis (PCA) and Bayesian population assignment. PCA was carried out using the R package adegenet v.2.0.1 (Jombart, 2008). Bayesian assignment to genetic clusters (populations) was carried out using the software STRUCTURE v2.3 (Pritchard et al., 2000). In the latter, we were interested in the number of genetic clusters identified, but also if the SNPs could be used to assign lynx to their correct source cluster. To examine this, we first conducted the STRUCTURE analysis on samples $(N=99)$ from naturally occurring populations and then tested whether samples from reintroduced populations (Croatia, Slovenia; $N=20$ ) would be assigned to the genetic cluster corresponding to their source population, i.e. the population from which lynx had been translocated to establish the reintroduced populations. First, for all 99 lynx from naturally occurring populations, we ran 10 replicates for values of $K$ (inferred number of genetic clusters) from 1 to 8 for 600,000 iteration steps, the first 150,000 of which were discarded as burn-in, and allowing for correlated allele frequencies in the admixture model (Falush et al., 2003). The most likely number of genetic clusters $(K)$ was then determined following the $\Delta K$-method (Evanno et al., 2005) implemented on the STRUCTUREHARVESTER website (http://taylorO.biology.ucla.edu/structureHarvester) (Earl \& vonHoldt, 2012). Using the inferred $K$, we then re-ran STRUCTURE with all lynx $(N=119)$ to check for correct cluster assignment. 
We tested for linkage disequilibrium (LD) and deviation from Hardy-Weinberg equilibrium (HWE) with GENEPOP (Raymond \& Rousset, 1995) in sampling localities and inferred genetic clusters; the R package LDheatmap (Shin et al., 2006) was used to visualize pairwise $r^{2}$ values.

\section{Applicability of baits for other taxa}

To assess the potential taxonomic breadth for which our cat CDS derived baits may be applicable, we queried our baits against the published genomes of other carnivore species using BLASTN: Iberian lynx (Lynx pardinus v1.0), cheetah (Acinonyx jubatus v1.0), leopard (Panthera pardus v1.0), tiger (Panthera tigris altaica v1.0), dog (Canis lupus familiaris v3.1), ferret (Mustela putorius furo v1.0), polar bear (Ursus maritimus v1.0), and giant panda (Ailuropoda melanoleuca v1.0). As sequence divergence between bait and target impacts enrichment (Vallender, 2011; Bi et al., 2012; Hedtke et al., 2013; Peñalba et al., 2014; Paijmans et al., 2016), we determined the sequence similarity between the baits and their corresponding best resulting hit in the queried genomes.

\section{RESULTS}

\section{Sequence and target-enrichment results}

The number of raw reads varied from 19,467,436 to 46,579,692 among the 26 lynx samples (mean: $28,094,688$ ), and the percentage of on-target sequences ranged from $19.6 \%$ to $29.5 \%$ (mean: $24.4 \%$; Table 1). As the target region corresponds to $0.026 \%$ of the cat genome (supplemental Table S2), and the lynx genome is roughly equivalent in size (Animal Genome Size Database, http://www.genomesize.com), the cross-species capture resulted in a greater than 900 -fold increase in ontarget sequences. 
For half of the CDS the enrichment was very successful: on average, $90 \%$ or more of the target region was covered at $\geq 15 \times$ depth (Fig. 2A). Across all samples, the majority of CDS (632 loci; $78.1 \%$ ) had a coverage of $\geq 15 \times$ for $50 \%$ or more of their lengths. Some CDS (61 loci; $7.5 \%$ ) were poorly enriched in all samples (less than $10 \%$ of target region $\geq 15 \times$ depth), among them 31 CDS were not enriched in any sample.

As expected, the inclusion of flanking sequences in the lynx CDS references (300 bp, both 5' and 3', see METHODS) increased the number of mapped sequences and improved coverage at CDS boundaries. This yielded between 16,701 and 30,309 more bases of the target with $\geq 15 \times$ depth (mean: 20,674 bases; $3.34 \%$ of the target region).

\section{Variation in $C D S$}

Of the 809 CDS analyzed, 61 were excluded due to insufficient data, 207 showed no variation among lynx samples (most probably due to poor coverage of CDS at $\geq 15 \times$ depth) and one showed no variation between lynx and cat. Three further CDS were no longer present in the newest build of the cat genome (v8.0). The remaining 537 CDS (66.4\% of CDS; length ranging from 961 to 5113 bp incl. flanking sequence) showed intraspecific variation, which consisted of 1186 SNPs and 109 InDels (Fig. 2B).

Use of the Iberian lynx genome as reference (see METHODS) revealed fewer intraspecific variants among Eurasian lynx samples (993 SNPs and 66 InDels). Most of this difference in the number of variants detected reflects differences in the genome assemblies of the cat and the Iberian lynx (i.e. fewer CDS present in the genome assembly, less flanking sequence retrievable) - in other words, the variation detected using the Iberian lynx genome was mostly a subset of the variation detected using the cat genome. Variants detected only when using the Iberian lynx genome as reference were located in the flanking regions of 14 CDS (18 SNPs, 3 InDels), which were characterized by low or no coverage using 
the cat genome. Thus, although the Iberian lynx is a closer relative of the Eurasian lynx than is the domestic cat, the more complete genome assembly of the cat enabled us to retrieve more data about intraspecific variation in the Eurasian lynx.

\section{SNP-panel}

Out of the 686 candidate SNPs inside CDS (57.8\% of SNPs), we selected 144 for further evaluation (see METHODS for selection criteria). This number was then further reduced to 96 SNPs based on reliability for genotyping on the chosen platform (Fluidigm Corp., '96.96 Dynamic Array Chip for Genotyping'). Based on the distribution of the selected SNPs when projected onto the cat genome (Fig. 1C) and assuming a similar distribution in lynx, we estimated the average distance between SNPs on the same chromosome in the final set of 96 SNPs to be $17.55 \mathrm{Mb}$.

As indicated by the improved genotyping success (Fig. 3), the final set of 96 SNPs had less missing data or genotyping inconsistencies among sample replicates of the 26 lynx, than did the set of all 144 SNPs. Five out of the 26 diluted samples $(0.5 \mathrm{ng} / \mu \mathrm{L}$ of DNA), which approximated poor quality samples (e.g. non-invasively collected samples), had a high incidence of missing data (visible as outliers in Fig. 3 left and mid). We obtained complete or nearly complete genotypes for 93 of the 96 additional lynx samples (Fig. 3 right, mean genotyping success $=97 \%$ ). We found no significant linkage between loci (LD heatmap in suppl. Figure S2), and detected no deviation from Hardy-Weinberg equilibrium in sampling localities or inferred genetic clusters (below).

Cross-species tests resulted in low overall genotyping success, except for the domestic cat (upon which the cross-species capture was based), which generated data at 83 loci, out of which only five were heterozygous (6\%). The other prey species displayed signals at a far lower rate: roe deer at 13 loci, European red deer at 9 loci, European hare at 8 loci, house mouse at 2 loci, pine marten at 27 loci, and 
American mink at 24 loci. Thus, the most likely prey species (roe deer, red deer, hare, [Jobin et al., 2000; Belotti et al., 2014]) would not generate false positive lynx genotypes; for the domestic cat (and presumably also the wild cat Felis silvestris), species-identification using commonly employed mtDNA markers may be necessary to exclude false positive lynx genotypes.

\section{Identification of individuals}

All 96 SNPs of the final panel were polymorphic for the complete lynx dataset $(N=119)$, with a minor

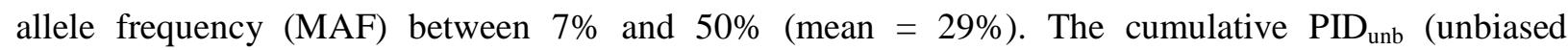
probability of identity) within geographic localities ranged from $7.13 \times 10^{-18}$ to $2.31 \times 10^{-34}$ (Table 2); and in the genetic clusters identified in the population structure analyses (below) from $3.54 \times 10^{-22}$ to $3.76 \times 10^{-}$

${ }^{34}$. The more conservative estimate of $\mathrm{PID}_{\text {sib }}$ (probability of identity given siblings) ranged from $1.53 \times 10^{-}$ ${ }^{8}$ to $3.39 \times 10^{-17}$ within geographic localities, and from $3.61 \times 10^{-11}$ to $5.12 \times 10^{-17}$ in genetic clusters (Table 2). As little as 24 loci were already sufficient to achieve a $\operatorname{PID}_{\text {sib }}<10^{-4}$, regardless of locality or genetic cluster (Table 2). When we examined the performance of SNPs to differentiate all 119 lynx using various subsets of loci (ranging from 10 to 90 loci; Fig. 4), we found that 60 SNPs were sufficient to differentiate all individuals in more than $98 \%$ of 10,000 random permutations of SNP loci (sampled without replacement). Thus, given the observed genotyping success rate (Fig. 3), our final 96 SNP-panel should perform very well for individual identification.

\section{Population structure inferences}

The PCA showed a clear separation of two distinct clusters along the first principle component axis (Fig. 5A), which explained $18.1 \%$ of variation. These two clusters corresponded to the Eurasian lynx subspecies Lynx lynx lynx (left) and L. l. carpathicus (right), which can also be differentiated using 
microsatellites (e.g. Ratkiewicz et al., 2014; Bull et al., 2016). Some substructure within subspecies is also apparent.

In STRUCTURE, the most likely number of genotypic clusters was $K=2$ (Fig. 5B, upper panel). However, the probability was also high for $K=5$ (supplemental Fig. S3). Since the Evanno method is designed to detect the highest hierarchical level of genetic structure (Evanno et al., 2005), we also considered $K=5$ (Fig. 5B lower panel).

Like in the PCA, the two inferred genotypic clusters $(K=2)$ corresponded to the two subspecies L. l. lynx (Fig. 5B, upper panel, yellow) and L. l. carpathicus (Fig. 5B, upper panel, blue). The lynx from the reintroduced populations in Croatia and Slovenia were assigned with high Q-values (0.75 - 0.99, mean 0.91) to the cluster of their source population (represented by Romanian and Slovakian samples).

The $K=5$ plot (Fig. 5B, lower panel) displayed a substructure in L. l. lynx: individuals from Estonia + Latvia, Poland, Russia + Finland, and Norway now formed their own clusters. There was also admixture between these clusters; most prominently from Russia + Finland into Estonia + Latvia. This extent of detected population genetic structure within L. l. lynx is similar to the one previously reported using microsatellites (Ratkiewicz et al., 2014). Again, all lynx from reintroduced populations were assigned with high Q-values $(0.73-0.98$, mean 0.88$)$ to the cluster of their source population.

\section{Applicability of baits in other taxa}

We found high sequence similarity between the cat CDS derived baits and their targets in four other felid species, Iberian lynx, cheetah, leopard and tiger (all four: median sequence divergence of 0.8\%; Fig. 6). As expected, sequence divergence between cat CDS derived baits and their targets in other, more distantly related carnivoran species was higher, with a median sequence divergence of $5.8 \%$ (Fig. 6). 


\section{DISCUSSION}

Our results show that cross-species capture of coding DNA sequences (CDS) can be used for SNP discovery in non-model organisms, and that a subset of the identified SNPs can be successfully implemented in a high throughput genotyping platform to accurately identify individuals and to infer population genetic structure of the species of interest.

Using publicly available genomic resources for model organisms, we were able to design baits for 100s of target CDS loci that were then enriched in our non-model study species, the Eurasian lynx Lynx lynx. We successfully surveyed intraspecific variation in L. lynx across its European range, and generated a large data set of SNPs inside CDS and their flanking regions. A large proportion of CDS had good or complete coverage of the target-region $(\geq 15 \times$ depth) and yielded 1186 SNP loci for downstream applications.

\section{Cross-species capture for SNP discovery}

Hybridization capture does not require an exact sequence match between bait and target for successful enrichment. While decreasing sequence similarity between bait and target reduces the efficiency of capture (Paijmans et al, 2016), successful enrichment has been reported for species with up to $40 \%$ sequence divergence ( $\mathrm{Li}$ et al., 2013). This 'mismatch tolerance' is utilized in cross-species capture, where baits designed using molecular data of one species are used to enrich complementary sequences in one or more other species. In interspecific studies, this yields comparable data (100s-1000s loci) across distantly related species (Lemmon et al., 2012; Faircloth et al., 2012; Bi et al., 2012).

Rather than generating comparable data across multiple species, we used cross-species capture to gain comparable data across many samples within the same species. In this manner, we generated high sequence coverage for a small portion of the nuclear genome - the targeted CDS - across all samples. 
This portion of the genome was then screened for variation in the form of SNPs, a subset of which was then used to design the SNP-panel.

Hybridization capture is scalable, with the number of targets determined by bait design. To ensure the recovery of sufficient variation for the development of a 96 SNP-panel, we chose to target several hundred CDS spread throughout the genome. However, considering that we far exceeded the number of SNPs required for the development of a 96 SNP-panel, a smaller number of targets would have been sufficient. For the purpose of developing a SNP-panel of similar size, we would still recommend a number of target loci exceeding what is practical using DIY protocols (e.g. 51 loci, Peñalba et al., 2014). Especially, since the per-sample costs of using custom baits (e.g. from MYcroarray) can be reduced by using smaller reaction volumes in combination with a dilution of synthetic RNA-baits (this study; Li et al., 2013; Cruz-Dávalos et al., 2016) and by pooling barcoded libraries of multiple samples prior to hybridization (e.g. Portik et al., 2016; Cruz-Dávalos et al., 2016). In addition, lowering the sequence depth requirement for SNP calling permits pooling of more samples during sequencing and can further reduce costs (e.g. $\geq 8 \times$ depth, Lim \& Braun, 2016). Considering our results, and setting a limit of one SNP per target locus for the SNP-panel, we would recommend a minimum of 250 target loci (400 bp or longer) for SNP discovery using an approach like the one described here.

The increasing availability of genomic resources for non-model organisms, particularly annotated genomes and transcriptomes, improves the chances of finding species for bait-design that are not too distantly related to the target species. While successful enrichment of sequences has been reported for 'bait species' with very high divergence times from the target species (up to $~ 250$ million years; Li et al., 2013; Hedtke et al., 2013), several studies examining capture efficiency on a per-target basis (e.g. per exon) have observed a drop in capture efficiency when sequence divergence between bait and target reaches 5-10\% (Vallender, 2011; Bi et al., 2012; Peñalba et al., 2014; Bragg et al., 2016). Such a drop in the performance of enrichment would require greater sequencing effort to achieve good sequence 
coverage of target loci and would thus increase costs for surveying intraspecific variation using crossspecies capture. For this reason, we examined the taxonomic breadth at which the baits used here should still perform well. The relatively limited sequence divergence between the cat CDS derived baits and their targets in other carnivorans with published genomes (Iberian lynx, cheetah, leopard, tiger, domestic dog, ferret, polar bear and giant panda; Fig. 6), suggests a relatively broad utility of these baits. This in silico assessment showed that the felid species (Iberian lynx, cheetah, leopard and tiger) have low sequence divergence between bait and target, suggesting that the baits we used to enrich CDS in lynx ought to perform very well for other species in the family Felidae (consisting of 14 genera and 37 species) that diverged approximately 11 million years ago (Johnson et al. 2006; Li et al., 2016). Unfortunately, except for felids, no other species of the feliform suborder of carnivorans have a published genome (yet); this suborder includes mongooses, meerkats, hyenas, linsangs, civets, and others (6 families without Felidae, with 83 species) that diverged from felids approximately 38 million years ago (Eizirik et al., 2010). Several species of the more distantly related caniform suborder of carnivorans do have a published genome (domestic dog, ferret, polar bear and giant panda). The caniform suborder (9 families, 161 species) diverged from the feliform suborder approximately 59 million years ago (Eizirik et al., 2010). These species appear to be at the limit of what could be considered suitable in terms of costeffective use of cross-species hybridization for surveying intraspecific variation (using this set of baits). While somewhat crude, this assessment suggests that this single set of baits is suitable to examine intraspecific variation in many species (across taxonomic families or even suborders) over quite substantial divergence times.

Because hybridization capture can be used on samples of poor quality (non-invasively collected material, Perry et al., 2010; archival material, McCormack et al., 2016; Lim \& Braun, 2016; ancient material, Carpenter et al., 2013; Enk et al., 2014) it is valuable for studies of taxa for which samples are difficult to obtain for genetic analyses (rare and elusive species, those in difficult to reach habitats, and degraded 
samples such as archival material). For example, non-invasively collected material can be incorporated in the SNP discovery process to cover portions of a species' distribution without having fresh tissue samples available. Or, archival and ancient samples can be added to existing data sets, providing information about historical (e.g. extinct) populations (Bi et al., 2014; Lim \& Braun, 2016).

\section{Genetic monitoring using SNPs in CDS}

The ability to accurately identify and differentiate individuals is central to population monitoring (Frankham et al., 2010). Thus, molecular markers used in non-invasive genetic monitoring must have sufficient power to differentiate individuals - even closely related individuals - and overcome the analytical difficulties often associated with non-invasively collected sample material, namely DNA extractions of low volume and low concentration that are characterized by varying levels of DNA degradation.

For our SNP-panel, we adopted a SNP typing platform, Fluidigm's Dynamic Array Chips, which has been successfully used to genotype SNPs in a range of non-invasively collected material, such as individual hair, faecal samples, and urine samples (Nussberger et al., 2014; Kraus et al., 2015). Using this platform, we observed a high genotyping success rate for $80 \%$ of samples with very low DNA concentrations $(0.5 \mathrm{ng} / \mu \mathrm{L})$, which we used in lieu of non-invasive samples. Conducting multiple replicates per sample is unproblematic, as only a limited amount of template is required per sample, and the costs of genotyping samples are relatively low (Kraus et al., 2015).

Regarding the identification of individuals, the 96 SNP format performed very well. This is in line with previous studies, which found that 40-100 SNP loci performed similarly well or better than the typical number (10-20) of microsatellite loci used for the purpose of individual identification and kinship analysis (e.g. Tokarska et al., 2009; Hauser et al., 2011; Gärke et al., 2012; Morin et al., 2012; Weinman 
et al., 2015; Kaiser et al., 2017). Here, we observed that a low number (23 or 24) of informative loci was more than sufficient to distinguish individuals of a given population. Our permutation test showed that even with a substantial number of locus drop-outs (up to 30-40\%), we were able to distinguish individuals, indicating that this 96 SNP-panel is robust enough for genotyping poor quality (e.g. noninvasively collected) samples.

The ability to make population structure inferences and to assign individuals to populations is an important component of genetic population monitoring, providing information about animal movements and potential gene flow, the impact of habitat fragmentation, degree of inbreeding, and other population parameters (Frankham et al., 2010). In past years, there has been increasing evidence for the suitability of genic SNPs to ascertain population membership of individuals (Freamo et al., 2011; Defaveri et al., 2013; Oliveira et al., 2015; Zhan et al., 2015; Elbers et al., 2016). This has been examined by itself and also in comparison with both traditionally used microsatellite markers (e.g. Defaveri et al., 2013; Elbers et al., 2016) and non-genic SNPs (e.g. Defaveri et al., 2013; Zhan et al., 2015; Elbers et al., 2016); in all cases, genic SNPs performed equally well or better than alternative markers.

Using our CDS derived SNPs we were able to unambiguously delineate the two Eurasian lynx subspecies in our sample set (L. l. lynx and L. l. carpathicus). Within L. l. lynx, which dominated our sample set, further levels of population structure could be resolved. This structure was congruent with the one detected in a larger sample set $(N=298)$ that had been genotyped at 13 microsatellite loci (Ratkiewicz et al., 2014). Similarly, the extent and direction of introgression detected using our 96 SNPpanel mirrored that observed using microsatellites in the aforementioned study. Lastly, using our 96 SNP-panel we were also able to accurately assign individuals from reintroduced populations to the genetic cluster of their source population. The 96 SNP-panel presented here thus appears more than suitable for the genetic monitoring of Eurasian lynx across their European range considering the higher 
potential for automation of SNP genotyping, better collaboration possibilities, and cost reduction potential (Kraus et al., 2015).

\section{CONCLUSION}

There is growing evidence for the utility of genic SNPs for the genetic monitoring of populations. As demonstrated here, hybridization capture coupled with high-throughput sequencing is well suited for acquiring information regarding such intraspecific variation, even in cases where study species lack genomic resources. With the increasing availability of genomic resources for non-model species, this kind of approach will become more broadly applicable - even though cross-species capture already shows potential to work across substantial divergence times (e.g. Li et al., 2013; Hedtke et al., 2013; Bragg et al., 2016).

Baits do of course not need to be explicitly designed with the aim of discovering SNPs for genetic monitoring purposes. Thus, baits designed for other purposes (e.g. resolving taxonomic uncertainties, Yuan et al., 2016; identifying regulatory sequences, Yoshihara et al. 2016; identifying adaptive genes, Roffler et al. 2016; investigating loci linked to traits, Springer et al., 2015) can be used to screen samples from related species for intraspecific variation.

\section{ACKNOWLEDGEMENTS}

This research was funded by the Leibniz Association (SAW-2011-SGN-3) and by the Deutscher Akademischer Austauschdienst (A/11/93927). The license for lynx live-trapping and blood sampling in Poland was obtained from the National Ethics Committee for Animal Experiments (no. DB/KKE/PL110/2001) and the Local Ethics Committee for Animal Experiments at the Medical University of 
Białystok, Poland (no. 52/2007). Import of samples from Russia was licensed by CITES permission no 12RU000512.

\section{REFERENCES}

Abascal F, Corvelo A, Cruz F, Villanueva-Canas JL, Vlasova A, Marcet-Houben M ... Godoy JA (2016) Extreme genomic erosion after recurrent demographic bottlenecks in the highly endangered Iberian lynx. Genome Biology, 17, 251.

Belotti E, Kreisinger J, Romportl D, Heurich M, \& Bufka L (2014) Eurasian lynx hunting red deer: is there an influence of a winter enclosure system? European Journal of Wildlife Research, 60, 441-457.

Bi K, Vanderpool D, Singhal S, Linderoth T, Moritz C, Good JM (2012) Transcriptome-based exon capture enables highly cost-effective comparative genomic data collection at moderate evolutionary scales. BMC Genomics, 13, 403.

Bi K, Linderoth T, Vanderpool D, Good JM, Nielsen R, \& Moritz C (2014) Unlocking the vault: next generation museum population genomics. Molecular Ecology, 22, 6018-6032.

Bolger AM, Lohse M, Usadel B (2014) Trimmomatic: a flexible trimmer for Illumina sequence data. Bioinformatics, 30, 2114-2120.

Bragg JG, Potter S, Bi K, \& Moritz C (2016) Exon capture phylogenomics: efficacy across scales of divergence. Molecular Ecology Resources, 16, 1059-1068.

Bull J, Heurich M, Saveljev A, Schmidt K, Fickel J, \& Förster D (2016) The effect of reintroduction on the genetic variability in Eurasian lynx populations: the cases of Bohemian-Bavarian and VosgesPalatinian populations. Conservation Genetics, 17, 1229-1234.

Carpenter ML, Buenrostro JD, Valdiosera C, Schroeder H, Allentoft ME, Sikora M ... Bustamante CD (2013) Pulling out the 1\%: whole-genome capture for the targeted enrichment of ancient DNA sequencing libraries. The American Journal of Human Genetics, 93, 852-864.

Cruz-Dávalos DI, Llamas B, Gaunitz C, Fages A, Gamba C, Soubrier J ... Orlando L (2016) Experimental conditions improving in-solution target enrichment for ancient DNA. Molecular Ecology Resources, early view, doi:10.1111/1755-0998.12595

DeFaveri J, Viitaniemi H, Leder E, \& Merila J (2013) Characterizing genic and nongenic molecular markers: comparison of microsatellites and SNPs. Molecular Ecology Resources, 13, 377-392.

Earl DA, vonHoldt BM (2012) STRUCTURE HARVESTER: a website and program for visualizing STRUCTURE output and implementing the Evanno method. Conservation Genetics Resources, 4, 359-361.

Eizirik E, Murphy WJ, Koepfli KP, Johnson WE, Dragoo JW, Wayne RK, \& O’Brien SJ (2010) Pattern and timing of diversification of the mammalian order Carnivora inferred from multiple nuclear gene sequences. Molecular Phylogenetics and Evolution, 56, 49-63.

Elbers JP, Clostio RW, \& Taylor SS (2016) Population genetic inferences using immune gene SNPs mirror patterns inferred by microsatellites. Molecular Ecology Resources, early view, doi: 10.1111/1755-0998.12591

Enk JM, Devault AM, Kuch M, Murgha YE, Rouillard J-M, \& Poinar HN (2014) Ancient whole genome enrichment using baits built from modern DNA. Molecular Biology and Evolution, 31, 1292-1294.

Evanno G, Regnaut S, \& Goudet J (2005) Detecting the number of clusters of individuals using the software STRUCTURE: a simulation study. Molecular Ecology, 14, 2611-2620. 
Faircloth BC, McCormack JE, Crawford NG, Harvey MG, Brumfield RT, \& Glenn TC (2012) Ultraconserved elements anchor thousands of genetic markers spanning multiple evolutionary timescales. Systematic Biology, 61, 717-726.

Falush D, Stephens M, \& Pritchard JK (2003) Inference of Population Structure Using Multilocus Genotype Data: Linked Loci and Correlated Allele Frequencies. Genetics, 164, 1567-1587.

Fortes GG, \& Paijmans JLA (2015) Analysis of whole mitogenomes from ancient samples. In: Whole genome amplification. Methods in Molecular Biology (ed. Kroneis T). Humana Press, New York, USA.

Frankham R, Ballou JD, \& Briscoe DA (2010) Introduction to conservation genetics (2 ${ }^{\text {nd }}$ ed.). Cambridge University Press, Cambridge, UK.

Freamo H, O'Reilly P, Berg PR, Lien S, \& Boulding EG (2011) Outlier SNPs show more genetic structure between two Bay of Fundy metapopulations of Atlantic salmon than do neutral SNPs. Molecular Ecology Resources, 11, 254-267.

Gärke C, Ytournel F, Bed'hom B, Gut I, Lathrop M, Weigend S, \& Simianer H (2012) Comparison of SNPs and microsatellites for assessing the genetic structure of chicken populations. Animal Genetics, 43, 419-428.

Gasc C, Peyretaillade E, \& Peyret P (2016) Sequence capture by hybridization to explore modern and ancient genomic diversity in model and nonmodel organisms. Nucleic Acid Research, 44, 4504-4518.

Hauser L, Baird M, Hilborn RAY, Seeb LW, \& Seeb JE (2011) An empirical comparison of SNPs and microsatellites for parentage and kinship assignment in a wild sockeye salmon (Oncorhynchus nerka) population. Molecular Ecology Resources, 11, 150-161.

Hedtke SM, Morgan MJ, Cannatella DC, \& Hillis DM (2013) Targeted enrichment: maximizing orthologous gene comparisons across deep evolutionary time. PLoS ONE, 8, e67908.

Jobin A, Molinari P, \& Breitenmoser U (2000). Prey spectrum, prey preference and consumption rates of Eurasian lynx in the Swiss Jura Mountains. Acta Theriologica, 45, 243-252.

Jombart T. (2008) adegenet: a R package for the multivariate analysis of genetic markers. Bioinformatics, 24, 1403-1405.

Kaiser SA, Taylor SA, Chen N, Sillet TS, Bondra ER, \& Webster MS (2017) A comparative assessment of SNP and microsatellite markers for assigning parentage in a socially monogamous bird. Molecular Ecology Resources, 17, 183-193.

Kraus RHS, VonHoldt B, Cocchiararo B, Harms V, Bayerl H, Kühn H ... Nowak C (2015) A singlenucleotide polymorphism-based approach for rapid and cost-effective genetic wolf monitoring in Europe based on non-invasively collected samples. Molecular Ecology Resources, 15, 295-305.

Lemmon AR, Emme SA, \& Lemmon EM (2012) Anchored hybrid enrichment for massively highthroughput phylogenomics. Systematic Biology, 61, 727-744.

Li H, \& Durbin R (2009) Fast and accurate short read alignment with Burrows-Wheeler transform. Bioinformatics, 25, 1754-1760.

Li C, Riethoven JJ, \& Naylor GJ (2012) EvolMarkers: a database for mining exon and intron markers for evolution, ecology and conservation studies. Molecular Ecology Resources, 12, 967-971.

Li C, Hofreiter M, Straube N, Corrigan S, \& Naylor GJ (2013) Capturing protein-coding genes across highly divergent species. BioTechniques, 54, 321-326.

Lim HC, \& Braun MJ (2016) High-throughput SNP genotyping of historical and modern samples of five bird species via sequence capture of ultraconserved elements. Molecular Ecology Resources, 16, 1204-1223.

Magoč T, \& Salzberg SL (2011) FLASH: fast length adjustment of short reads to improve genome assemblies. Bioinformatics, 27, 2957-2963.

Maricic T, Whitten M, \& Pääbo S (2010) Multiplexed DNA sequence capture of mitochondrial genomes using PCR products. PLoS One, 5, e14004. 
Martin M (2011) Cutadapt removes adapter sequences from highthroughput sequencing reads. EMBnet. Journal, 17, 10-12.

McCormack JE, Tsai WLE, \& Faircloth BC (2016) Sequence capture of ultraconserved elements from bird museum specimens. Molecular Ecology Resources, 16, 1189-1203.

Meyer M, \& Kircher M (2010) Illumina sequencing library preparation for highly multiplexed target capture and sequencing. Cold Spring Harbor Protocols, 2010(6), doi:10.1101/pdb.prot5448.

Morin PA, Archer FI, Pease VL, Hancock-Hanser BL, Robertson KM, Huebinger RM ... Taylor BL (2012) An empirical comparison of SNPs and microsatellites for population structure, assignment, and demographic analyses of bowhead whale populations. Endangered Species Research, 19, 129147.

Nussberger B, Wandeler P, \& Camenisch C (2014) A SNP chip to detect introgression in wildcats allows accurate genotyping of low quality samples. European Journal of Wildlife Research, 60, 405-410.

Oliveira R, Randi E, Mattucci F, Kurushima JD, Lyons LA, \& Alves PC (2015) Toward a genome-wide approach for detecting hybrids: informative SNPs to detect introgression between domestic cats and European wildcats (Felis silvestris). Heredity, 115, 195-205.

Paijmans JLA, Gilbert MTP, \& Hofreiter M (2013) Mitogenomic analyses from ancient DNA. Molecular Phylogenetics and Evolution, 69, 404-416.

Paijmans JLA, Fickel J, Courtiol A, Hofreiter M, \& Förster D (2016) Impact of enrichment conditions on cross-species capture of fresh and degraded DNA. Molecular Ecology Resources, 16, 42-55

Peñalba JV, Smith LL, Tonione MA, Sass C, Hykin SM, Skipwith PL ... Moritz C (2014) Sequence capture using PCR-generated probes: a cost-effective method of targeted highthroughput sequencing for nonmodel organisms. Molecular Ecology Resources, 14, 1000-1010.

Perry GH, Marioni JC, Melsted P, \& Gilad Y (2010) Genomic-scale capture and sequencing of endogenous DNA from feces. Molecular Ecology, 19, 5332-5344.

Portik DM, Smith LL, \& Bi K (2016) An evaluation of transcriptome-based exon capture for frog phylogenomics across multiple scales of divergence (Class: Amphibia, Order: Anura). Molecular Ecology Resources, 16, 1069-1083

Pritchard JK, Stephens M, \& Donnelly P (2000) Inference of population structure using multilocus genotype data. Genetics, 155, 945-959.

Prum RO, Berv JS, Dornburg A, Field DJ, Townsend JP, Lemmon EM, \& Lemmon AR (2015) A comprehensive phylogeny of birds (Aves) using targeted next-generation DNA sequencing. Nature 526, 569-573.

Ratkiewicz M, Matosiuk M, Saveljev AP, Sidorovich V, Ozolins J, Männil P ... Schmidt K (2014) Long-range gene flow and the effects of climatic and ecological factors on genetic structuring in a large, solitary carnivore: the Eurasian lynx. PLoS ONE, 9, e115160.

Roffler GH, Amish SJ, Smith S, Cosart T, Kardos M, Schwartz MK, \& Luikart G (2016) SNP discovery in candidate adaptive genes using exon capture in a free-ranging alpine ungulate. Molecular Ecology Resources, 16, 1147-1164.

Schubert M, Ginolhac A, Lindgreen S, Thompson JF, Al-Rasheid KAS, Willerslev E ... Orlando L (2012) Improving ancient DNA read mapping against modern reference genomes. BMC Genomics, 13, 178.

Shin JH, Blay S, McNeney B, \& Graham J (2006) LDheatmap: an R function for graphical display of pairwise linkage disequilibria between single nucleotide polymorphisms. Journal of Statistical Software, 16, 1-10.

Springer MS, Signore AV, Paijmans JLA, Vélez-Juarbe J, Domning DP, Bauer CE ... Campbell KL (2015) Interordinal gene capture, the phylogenetic position of Steller's sea cow based on molecular and morphological data, and the macroevolutionary history of Sirenia. Molecular Phylogenetics and Evolution, 91, 178-193. 
Templeton JEL, Brotherton PM, Llamas B, Soubrier J, Haak W, Cooper A, \& Austin JJ (2013) DNA capture and next-generation sequencing can recover whole mitochondrial genomes from highly degraded samples for human identification. Investigative Genetics, 4, 26.

Tokarska M, Marshall T, Kowalczyk R, Wójcik JM, Pertoldi C, Kristensen TN ... Bendixen C (2009) Effectiveness of microsatellite and SNP markers for parentage and identity analysis in species with low genetic diversity: the case of European bison. Heredity, 103, 326-332.

Valière N (2002) GIMLET: a computer program for analysing genetic individual identification data. Molecular Ecology Notes, 2, 377-379.

Vallender EJ (2011) Expanding whole exome resequencing into nonhuman primates. Genome Biology, 12, R87.

Von Arx M, Breitenmoser-Würsten C, Zimmermann F, \& Breitenmoser U (2004) Status and conservation of the Eurasian lynx (Lynx lynx) in 2001. Muri: KORA Bericht no $19.330 \mathrm{pp}$.

Weinman LR, Solomon JW, \& Rubenstein DR (2015) A comparison of single nucleotide polymorphism and microsatellite markers for analysis of parentage and kinship in a cooperatively breeding bird. Molecular Ecology Resources, 15, 502-511.

Yoshihara M, Saito D, Sato T, Ohara O, Kuramoto T, \& Suyama M (2016) Design and application of a target capture sequencing of exons and conserved noncoding sequences for the rat. BMC Genomics, 17, 593.

Yuan H, Jiang J, Jiménez FA, Hoberg EP, Cook JA, Galbreath KE, \& Li C (2016) Target gene enrichment in the cyclophyllidean cestodes, the most diverse group of tapeworms. Molecular Ecology Resources, 16, 1095-1106

Zhan X, Dixon A, Batbayar N, Bragin E, Ayas Z, Deutschova L ... Bruford MW (2015) Exonic versus intronic SNPs: contrasting roles in revealing the population genetic differentiation of a widespread bird species. Heredity, 114, 1-9.

\section{AUTHOR CONTRIBUTIONS}

D.W.F., R.H.S.K., H.B., R.K., C.N. and J.F. designed the study, K.S., A.P.S., and M.S. coordinated sample collection, D.W.F., J.B., M.A., J.L.A.P. carried out the experiments, D.W.F and D.L. analyzed and interpreted data, and D.W.F. and J.F. wrote the manuscript. All authors edited and approved the final manuscript.

\section{DATA ACCESSIBILITY}

Supplementary Tables S1 and S2 and Figures S1, S2 and S3 can be found online with the study; SNP genotypes, the final 96 SNP-panel assay, as well as an R script are deposited on DRYAD under doi: provided upon acceptance. Illumina sequences are deposited in the NCBI SRA under Accession no.: provided upon acceptance. 


\section{TABLE \& FIGURE LEGENDS}

Table 1. Sampling localities, sequencing results and cross-species capture results of 26 Eurasian lynx Lynx lynx.

Table 2. Probability of identity calculated for different subsets of the SNP data.

Figure 1. Schematic overview of study. (A) Schematic summary of the workflow, presenting both computational steps (light grey boxes) and laboratory steps (dark grey boxes). (B) Sampling localities of Eurasian lynx Lynx lynx across Europe. The current distribution of the species is shaded in blue. Large yellow circles represent samples used during the SNP discovery phase; small black dots represent additional samples genotyped using the developed 96 SNP-panel. (C) Schematic representation of the distribution of CDS targeted for enrichment (blue bars), projected onto cat chromosomes. The positions of the 96 SNP loci used for the SNP-panel are indicated by red diamonds. Black ovals show centromeres.

Figure 2. (A) Average recovery of the 809 CDS enriched in the 26 Eurasian lynx in the SNP discovery panel. For each CDS, the average percentage of the locus length covered at $\geq 15 \times$ depth is plotted (using $2 \%$ bins). $50 \%$ of CDS have above $90 \%$ sequence length coverage at $\geq 15 \times$ depth, indicated by the black vertical line. (B) Summary of the distribution of variation in CDS and flanking sequences, shown separately for SNPs and InDels.

Figure 3. Box plots displaying the genotyping success of Eurasian lynx samples at SNP loci: left, the discovery panel (26 lynx) for the initial set of 144 SNPs tested on the Fluidigm genotyping platform; middle, the final 96 SNP-panel genotyped on the discovery panel (26 lynx); and right, the final 96 SNPpanel on an additional 96 lynx (not part of discovery panel).

Figure 4. Comparison of the proportion of individuals recovered using different subsets of the SNP data, ranging from 10 to 90 loci. For each subset size 10,000 permutations (random selection of loci without replacement) were plotted; percentage values indicate the number of permutations in which all individuals in the dataset $(N=119)$ were identified. Scatter was added on the $\mathrm{x}$-axis for each subset to visualize density.

Figure 5. Population structure inferences using the 96 SNP-panel. (A) Principle component analysis (PCA); the first two principle component axes are plotted, with the geographic origin of samples indicated by colour. (B) Bayesian population assignment using STRUCTURE; results for $K=2$ (top) and $K=5$ (bottom) are displayed. Results for lynx from reintroduced populations (Croatia, Slovenia) are shown on the right.

Figure 6. Sequence similarity between the domestic cat CDS derived baits and their targets in other carnivorans with published genomes, including four felids (Iberian lynx, cheetah, leopard, and tiger) and four caniform carnivorans (dog, ferret, polar bear, and giant panda).

\section{Supporting Information}

Table S1. Additional lynx samples genotyped using the newly developed 96 SNP-panel.

Table S2. Summary of CDS markers. 
Figure S1. A comparison of the number of unique on-target sequences following one capture versus two consecutive captures, for five samples (see also Table 1). Shown are the number of on-target sequences $(\%)$, the number of duplicate sequences $(\%)$, and the fold-increase in the number of unique on-target sequences.

Figure S2. A 'LD heatmap' of pairwise $r^{2}$ values, for 92 SNP loci (loci with more than $5 \%$ missing data were removed).

Figure S3. Plot of $\Delta K$ values (STRUCTURE analysis) generated by STRUCTUREHARVESTER (Earl \& vonHoldt, 2012). 
Table 1. Sampling localities, sequencing results and cross-species capture results of 26 Eurasian lynx Lynx lynx

\begin{tabular}{|c|c|c|c|c|c|c|}
\hline $\begin{array}{l}\text { Sample } \\
\text { ID }\end{array}$ & Country & Population $^{\text {a }}$ & Raw reads & $\begin{array}{c}\text { Filtered } \\
\text { sequences }\end{array}$ & $\begin{array}{l}\text { On-target } \\
\text { reads }(\%)\end{array}$ & $\begin{array}{c}\% \text { of target } \\
\text { covered at } \\
15 x^{c}\end{array}$ \\
\hline Cro. $1 *$ & Croatia & reintroduced & 42321520 & 28717105 & 19.6 & 79.0 \\
\hline Cro. 2 & Croatia & reintroduced & 19467436 & 15178879 & 26.2 & 66.2 \\
\hline Est.1 & Estonia & natural & 20936570 & 15527441 & 24.6 & 80.3 \\
\hline Est. 2 & Estonia & natural & 25697066 & 17894280 & 25.6 & 81.9 \\
\hline Fin. 1 & Finland & natural & 22477300 & 13883204 & 27.6 & 82.4 \\
\hline Fin. 2 & Finland & natural & 22967626 & 16569199 & 24.7 & 82.8 \\
\hline Fin. 3 & Finland & natural & 22894258 & 16450208 & 24.2 & 63.4 \\
\hline Latv.1 & Latvia & natural & 22321270 & 17341768 & 23.6 & 77.7 \\
\hline Latv. 2 & Latvia & natural & 24394838 & 18562462 & 23.7 & 80.1 \\
\hline Latv. 3 & Latvia & natural & 21356226 & 16677724 & 20.8 & 81.6 \\
\hline Nor.1 & Norway & natural & 25157782 & 15829112 & 26.5 & 66.3 \\
\hline Nor.2 & Norway & natural & 25893728 & 18727706 & 24.1 & 82.9 \\
\hline Nor.3* & Norway & natural & 51194776 & 33117564 & 19.9 & 81.8 \\
\hline Pol.1 & Poland & natural & 36350048 & 22862227 & 27.0 & 83.1 \\
\hline Pol.2 & Poland & natural & 23139694 & 15996340 & 26.8 & 73.0 \\
\hline Rom.1 & Romania & natural & 23078140 & 17044276 & 27.4 & 83.1 \\
\hline Rom. 2 & Romania & natural & 24678618 & 16330922 & 29.5 & 83.2 \\
\hline Rom.3* & Romania & natural & 45479818 & 28824269 & 21.3 & 78.3 \\
\hline Rus.1 & Russia & natural & 28224284 & 20381841 & 22.9 & 80.6 \\
\hline Rus.2* & Russia & natural & 36384372 & 25422519 & 19.9 & 83.0 \\
\hline Rus.3 & Russia & natural & 24140510 & 18351015 & 23.1 & 81.0 \\
\hline Rus.4 & Russia & natural & 23737994 & 17874068 & 22.8 & 81.6 \\
\hline Svk.1 & Slovakia & natural & 21955584 & 16006621 & 29.2 & 82.8 \\
\hline Svk. 2 & Slovakia & natural & 25109422 & 18732792 & 26.8 & 83.7 \\
\hline Svn.1 & Slovenia & reintroduced & 24523316 & 18384101 & 24.9 & 85.1 \\
\hline \multirow[t]{2}{*}{ Svn. $2 *$} & Slovenia & reintroduced & 46579692 & 28828601 & 23.0 & 86.8 \\
\hline & & mean & 28094688 & 19596779 & 24.4 & 76.7 \\
\hline
\end{tabular}

* incl. data from single capture (see METHODS).

a 'natural' refers to autochthonous populations or populations that have recovered from population bottlenecks through natural means (i.e. without human assistance); 'reintroduced' populations are those established through human assistance (translocations, etc).

${ }^{\mathrm{b}}$ adapter and quality trimmed, merged sequences and sequences that could not be merged

${ }^{c}$ of the targeted $809 \mathrm{CDS}$ with a total length of $618547 \mathrm{bp}$ (i.e. not incl. flanking sequences). 
Table 2. Probability of identity calculated for different subsets of the SNP data

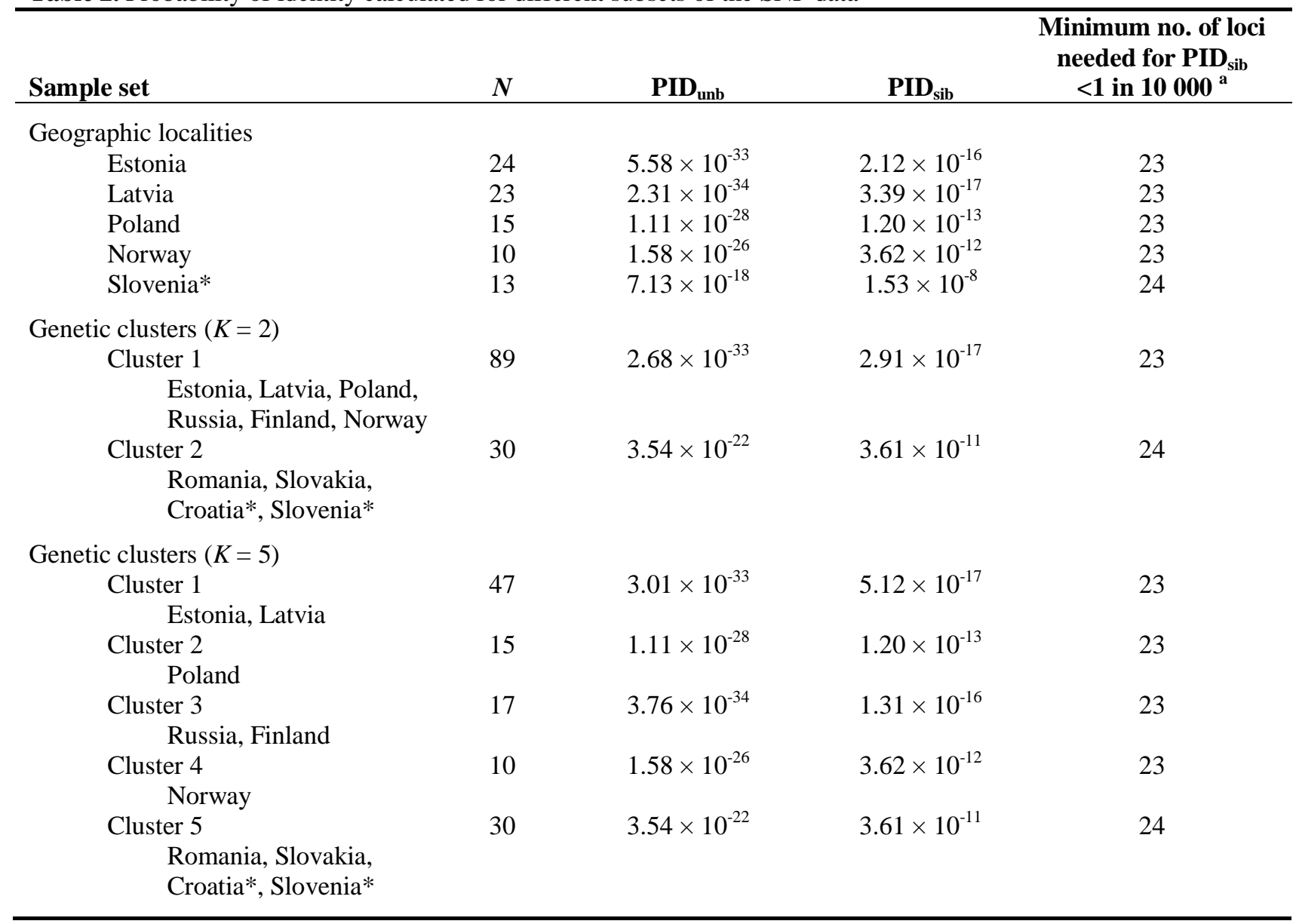

PID $_{\text {unb }}$ (unbiased probability of identity) and PID ${ }_{\text {sib }}$ (probability of identity given siblings) were calculated using GIMLETv1.3.3 (Valière, 2002)

* reintroduced population

${ }^{a}$ loci were ranked by informativeness (per sample set), and then the number of loci was determined for which PID $_{\text {sib }}$ was $<10^{-4}$. 
A.) Workflow

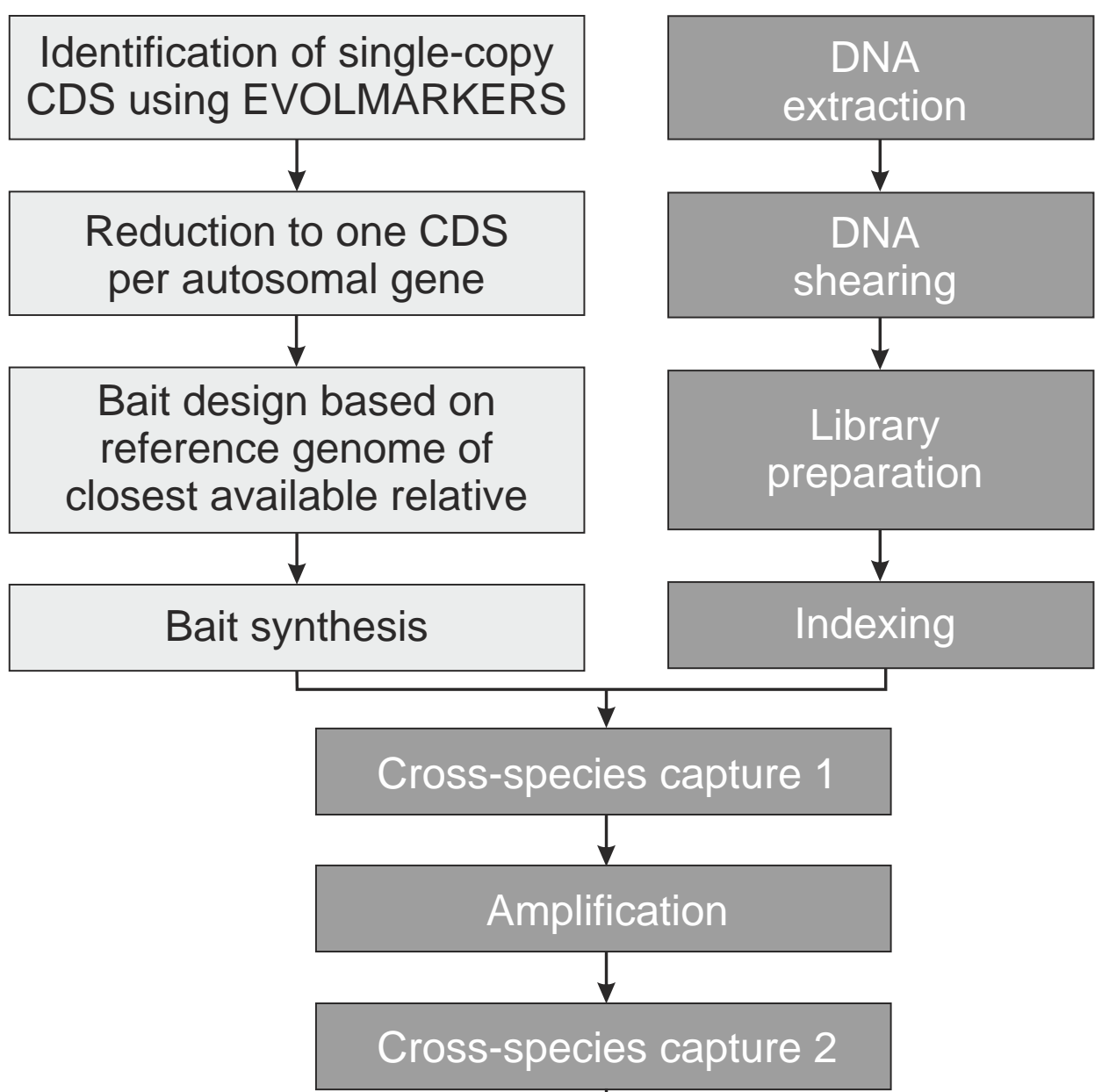

B.) Sampling

bioRxiv preprint doi: https://doi.org/10.1101 63659; this version posted July 14, 2017. The copyright holder for this preprint (which wass not

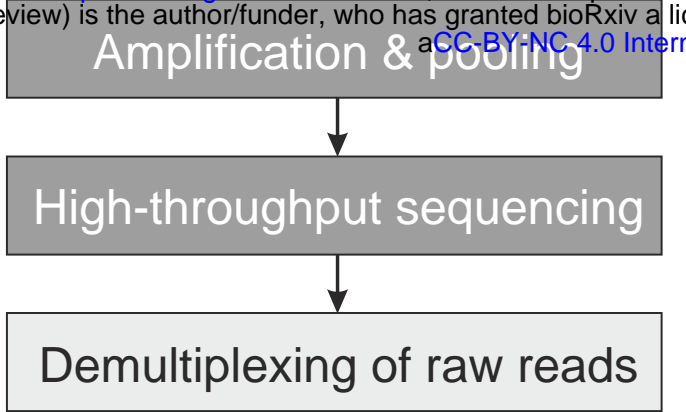

\begin{tabular}{|c|}
\hline \multicolumn{1}{|c|}{$\downarrow$} \\
Adapter trimming, quality \\
trimming \& merging of \\
overlapping paired-end reads
\end{tabular}

\section{C.) CDS mapped to cat genome}

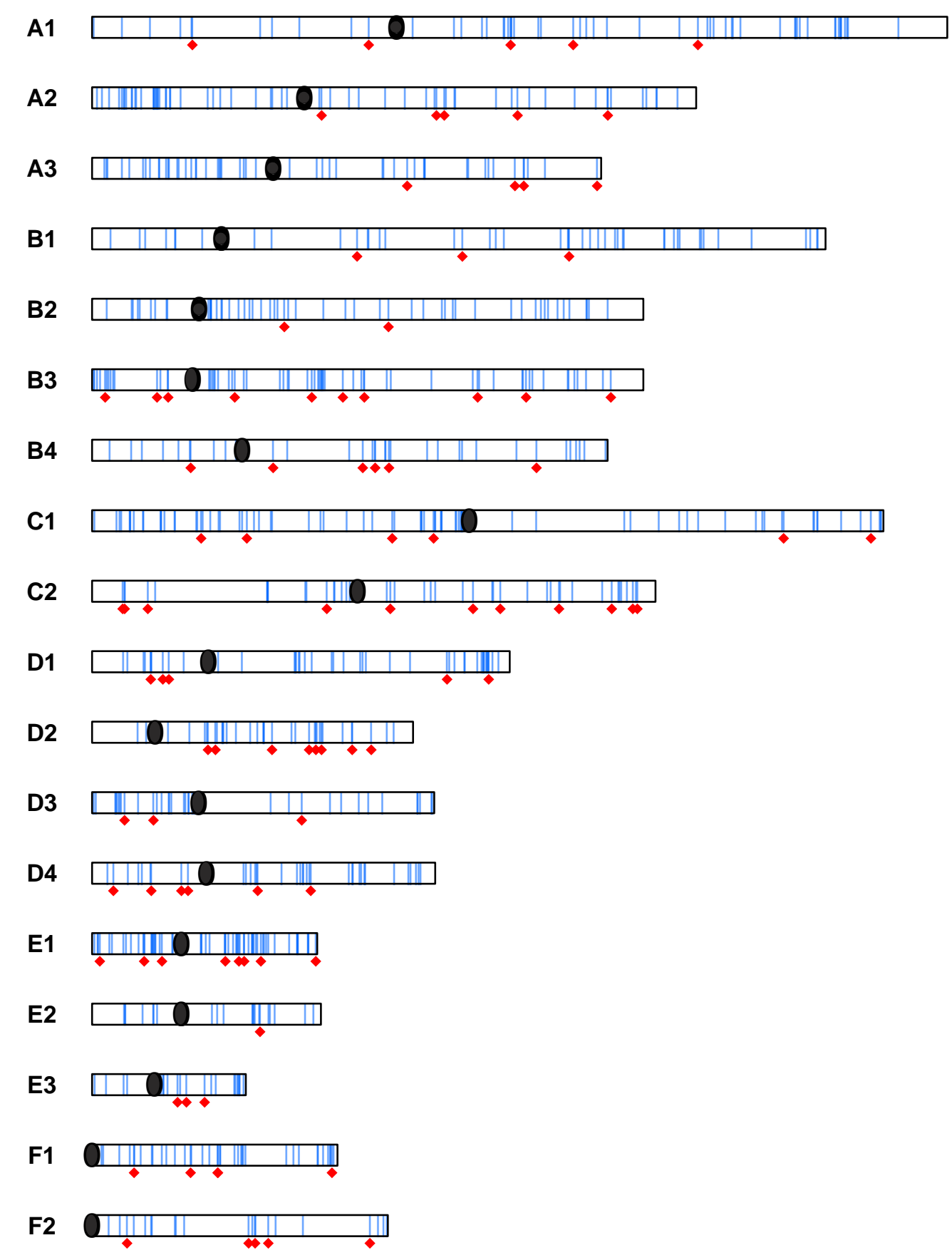

$0^{\circ}$.

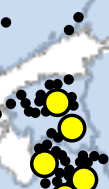

8
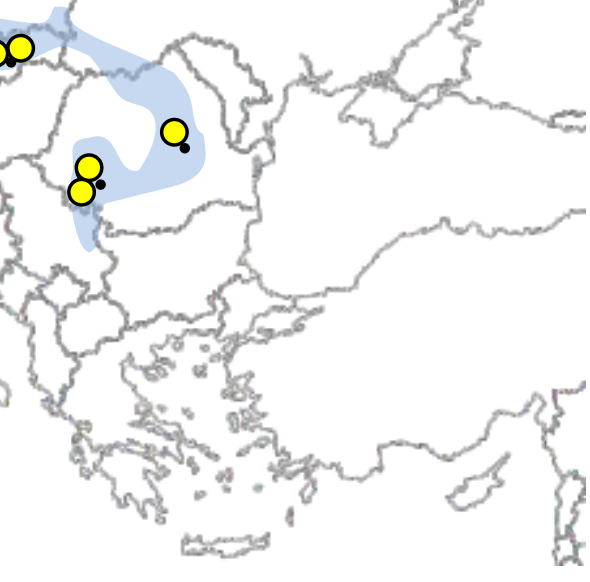


\section{A.) Enrichment of CDS}

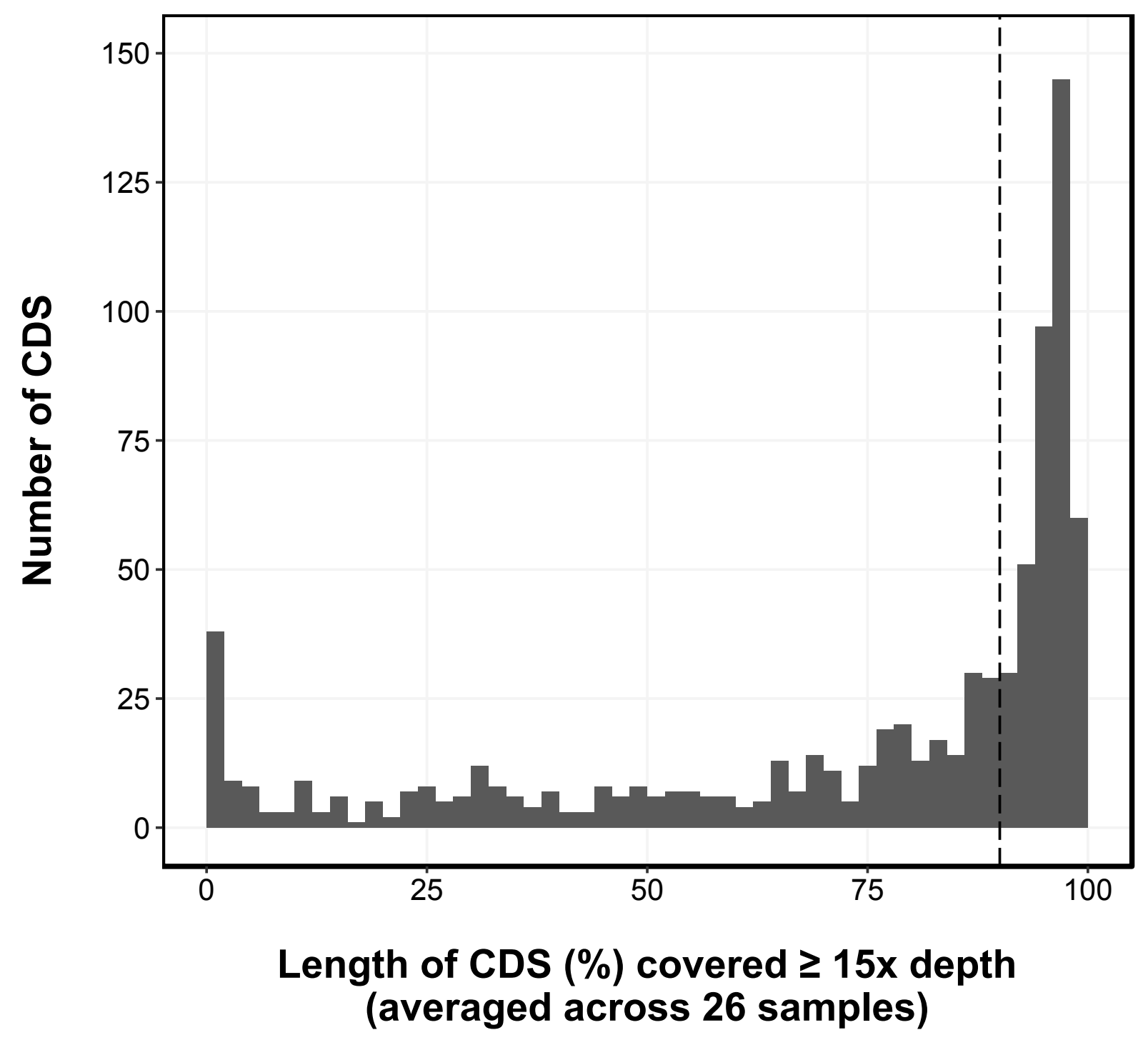

B.) Variants in CDS and flanking region

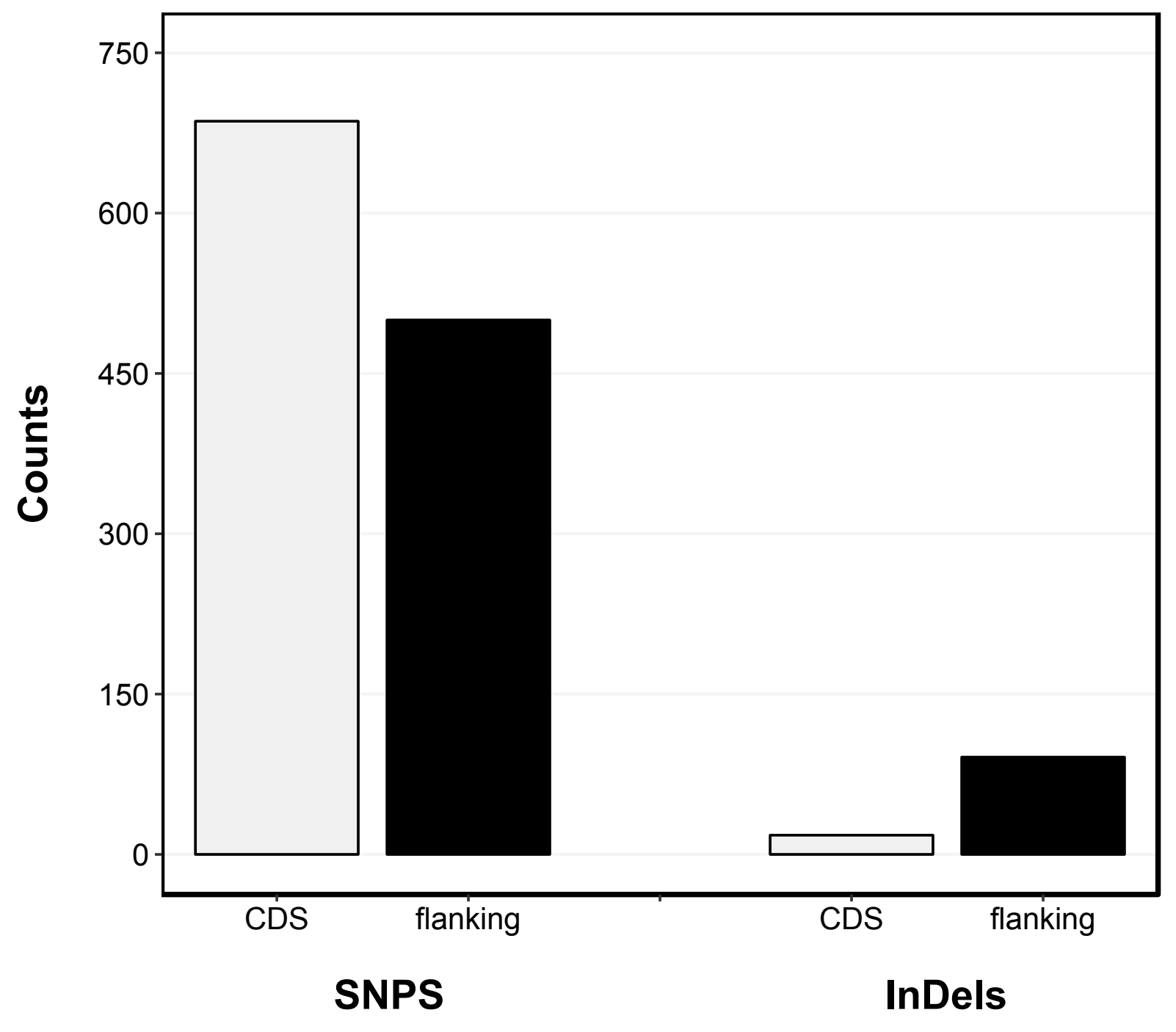




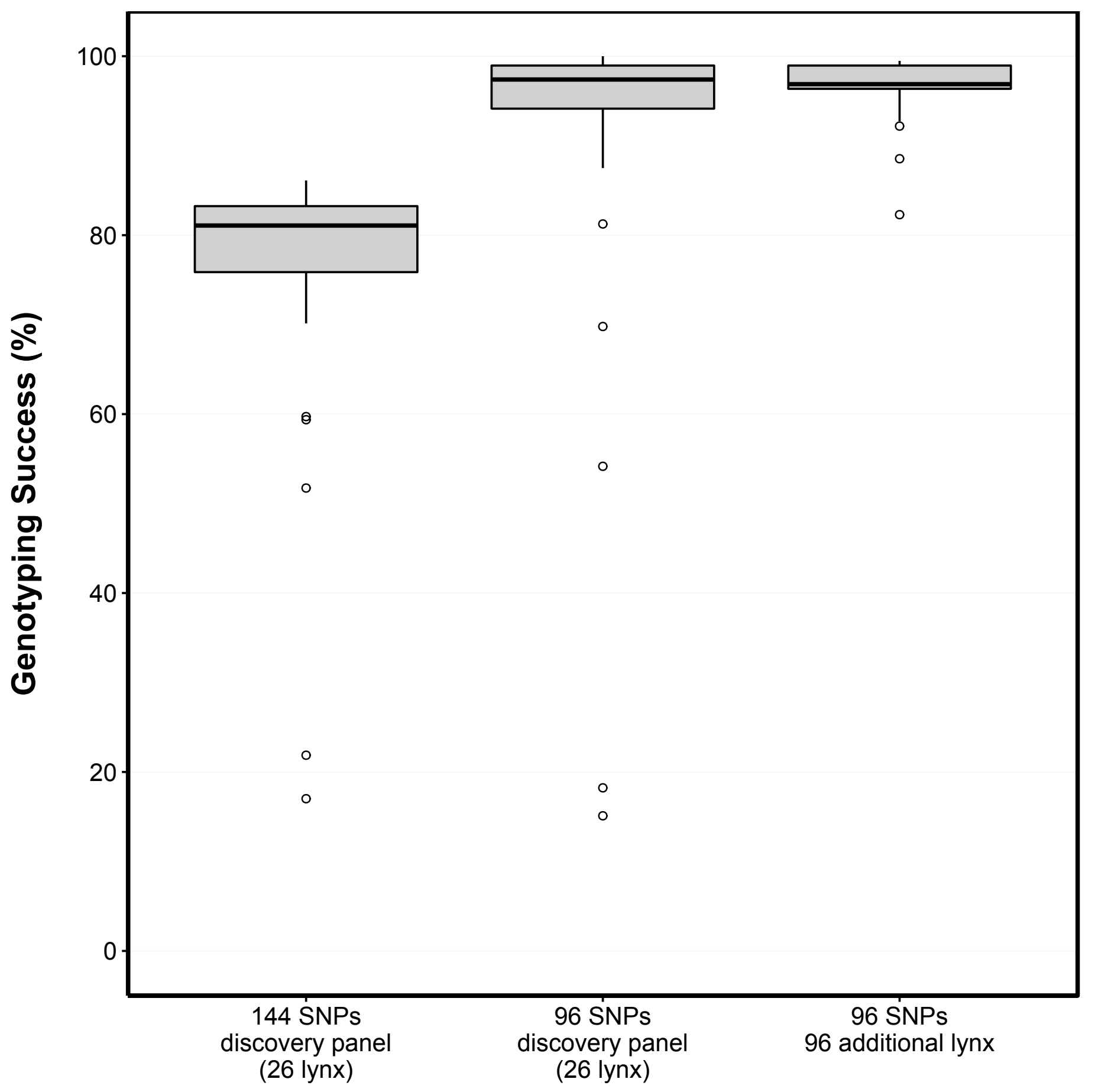




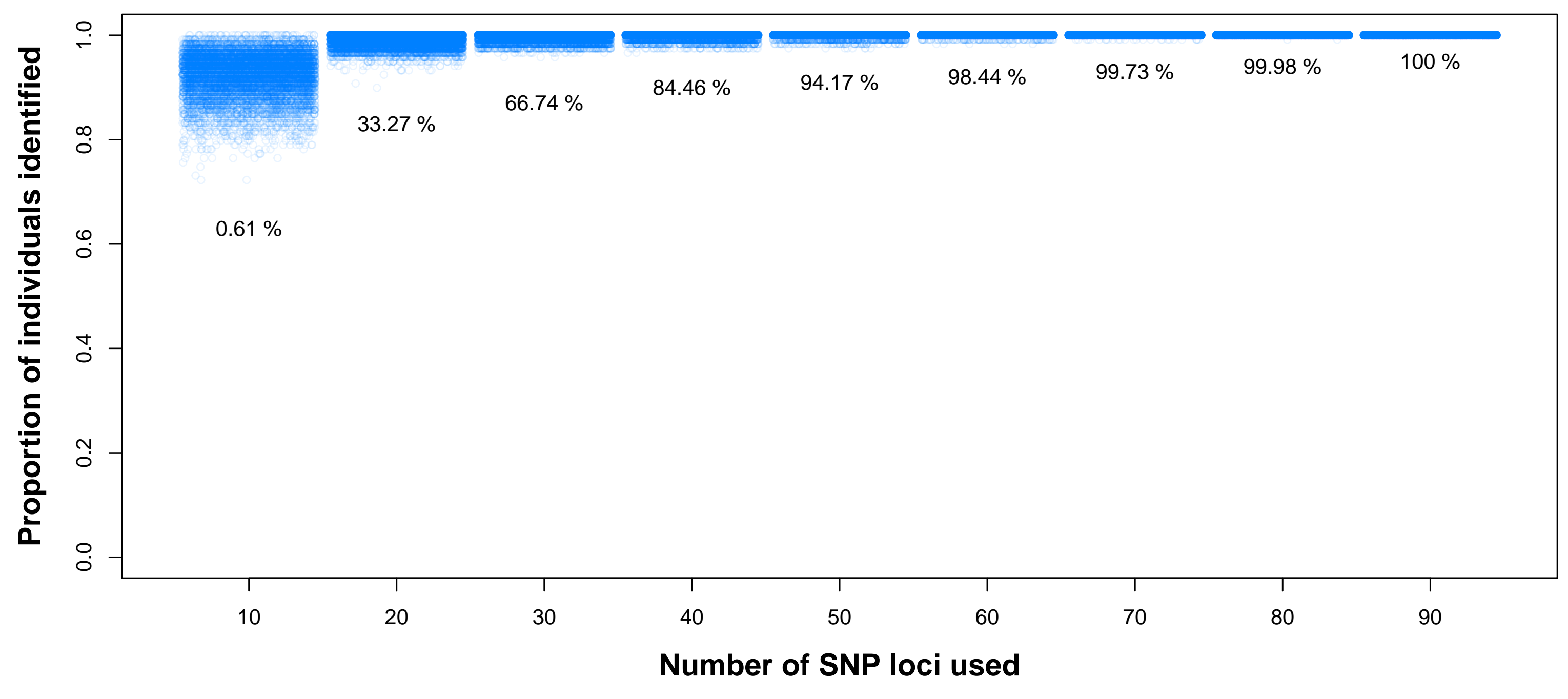


A.) PCA

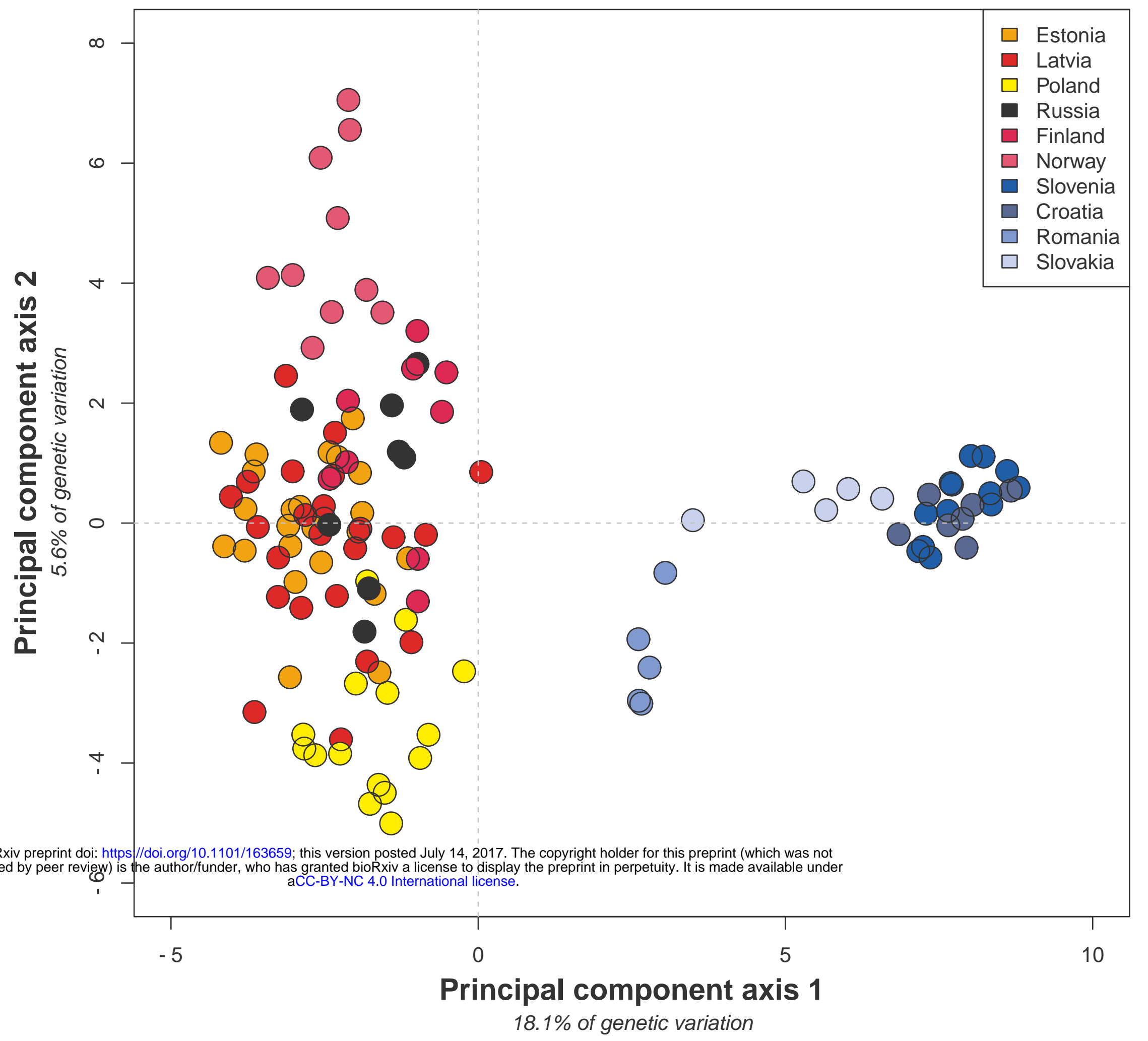

B.) Bayesian population assignment (STRUCTURE)
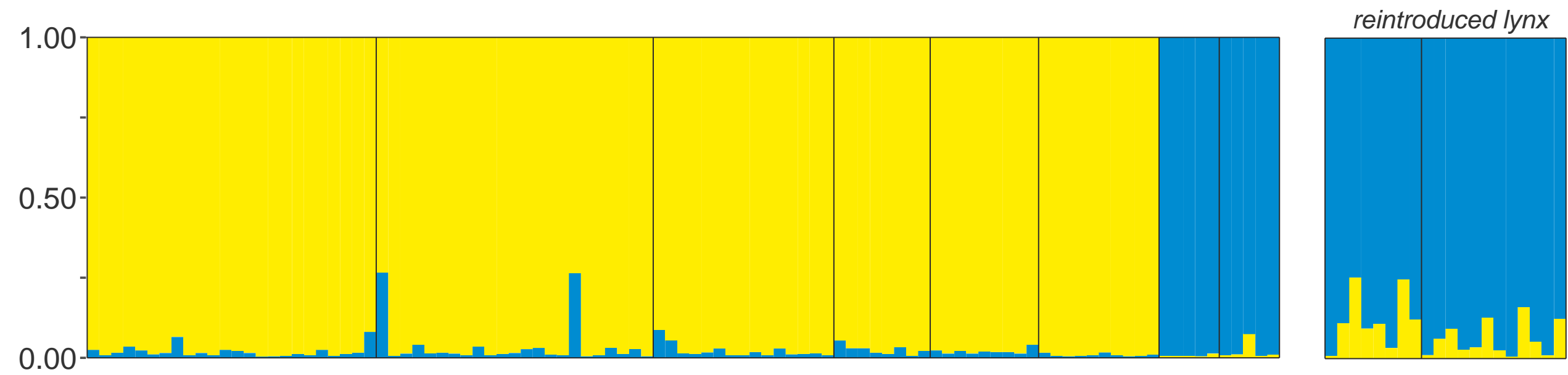

$K=2$
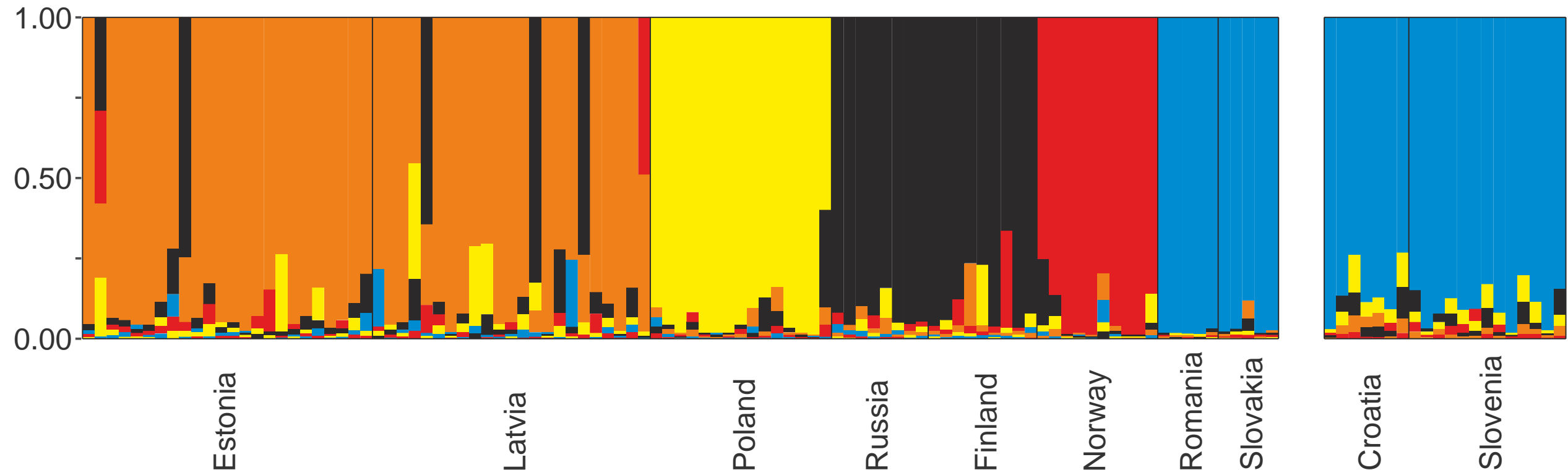

$K=5$

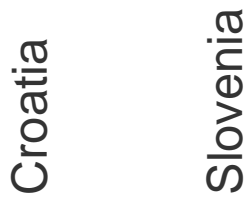




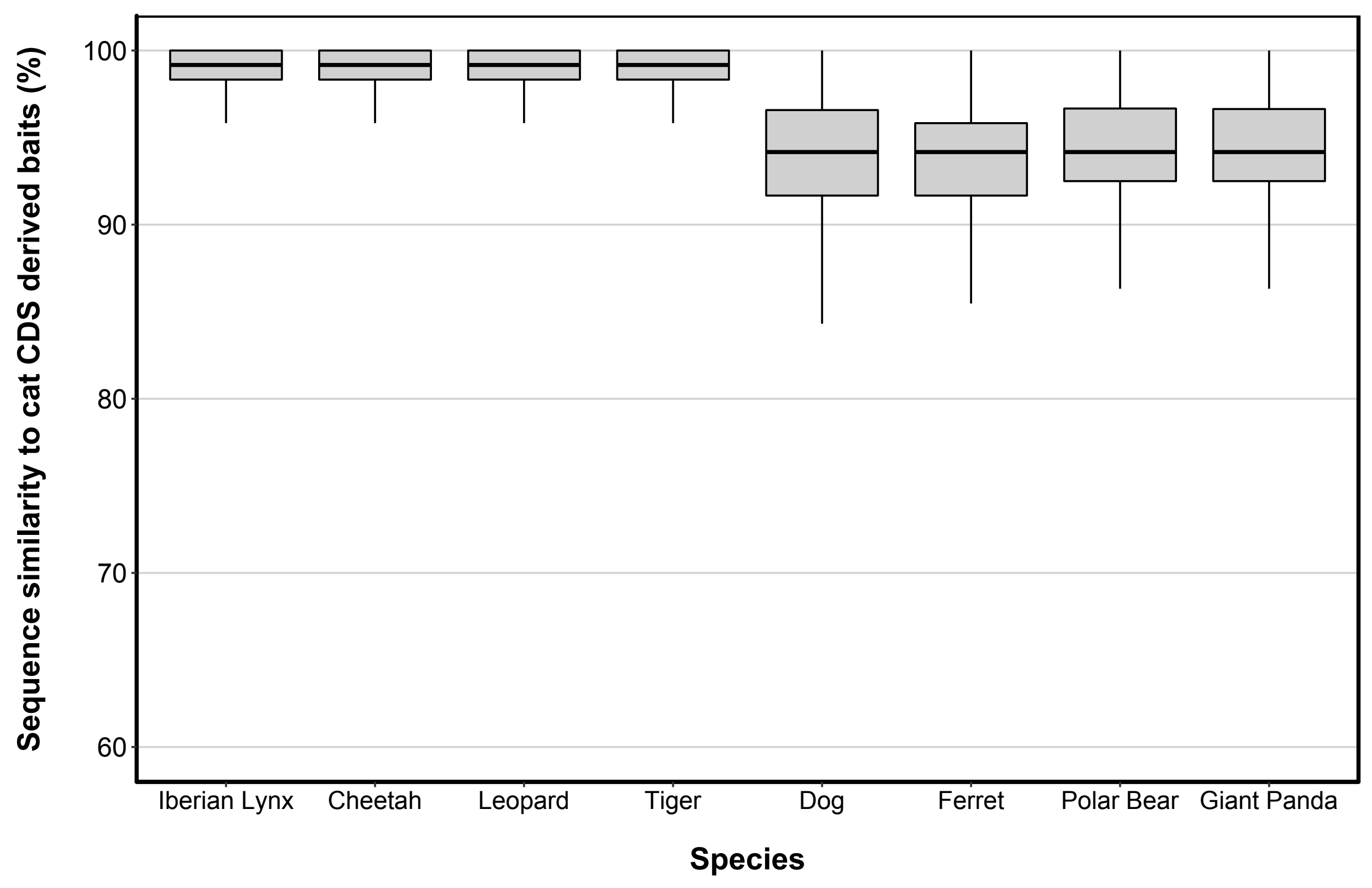

